\title{
Impfstoffe gegen neu auftretende Viren
}

\author{
Marylyn Addo $(\bowtie)$ \\ Sektion Infektiologie, Zentrum für Innere Medizin I, Universitätsklinikum \\ Hamburg-Eppendorf, Hamburg, Deutschland \\ m. addo@uke.de
}

Schlüsselwörter: Impfstoffe $\cdot$ Epidemien $\cdot$ Ebola $\cdot$ CEPI $\cdot$ COVID-19 · Adenovirus-Vektor $\cdot$ MVA-Vektor $\cdot$ m-RNA-Impfstoffe

\section{Ausbrüche neu aufgetretener Infektionskrankheiten}

Seit dem Jahr 2000 gab es eine Vielzahl von Ausbrüchen [1] und Epidemien, einschließlich der in der Folge genannten:

- ein SARS-Ausbruch:

2002-2003 China, Hongkong, Taiwan, Singapur, Vietnam.

- zwei Ausbrüche des Chikungunya-Fiebers:

2005 La Reunion und Mauritius, 2013-2014 Mittel- und Südamerika

- drei Epidemien des Zika-Virus:

2007 Mikronesien, 2013 pazifische Inselstaaten, 2015-2017 Mittel- und Südamerika

- vier große Cholera-Epidemien:

2008, 2018 Zimbabwe, 2010 Haiti, 2017 Jemen (größte Cholera Epidemie der Historie)

- die Schweinegrippe-Pandemie (H1N1):

2009-2010

- eine MERS-Epidemie:

2013-2015

- ein Ebola-Ausbruch in Westafrika:

2013-2016 Liberia, Sierra Leone und Guinea

- Gelbfieber-Ausbrüche:

2016-2017 Kongo und Angola und 2017 Brasilien

- eine Pest-Epidemie:

2017 Madagaskar

- ein Ausbruch des Lassa-Fiebers: 2018 Nigeria

- mehrere Masern-Epidemien im Kongo:

2018-2020

- drei Ebola-Ausbrüche im Kongo:

2018-2020

- COVID-19-Pandemie 
Diese Aufzählung verdeutlicht, wie häufig Epidemien auftreten. Eine Epidemie wird definiert als örtlich und zeitlich begrenztes gehäuftes Auftreten einer Erkrankung. In den letzten elf Jahren hat die Weltgesundheitsorganisation WHO immerhin sechs dieser Epidemien zu internationalen Gesundheitsnotfällen erklärt. Ein Notfallkomitee aus internationalen Experten stuft ein Ereignis als ein „Public Health Emergency of International Concern“ (PHEIC) ein, wenn eine Situation eintritt, die ,ernst, plötzlich, ungewöhnlich oder unerwartet“ ist und „Auswirkungen auf die öffentliche Gesundheit über die nationalen Grenzen des betroffenen Staates hinaus hat" sowie ,möglicherweise sofortige internationale Maßnahmen erfordert" [2]. Die PHEIC waren:

- Schweinegrippe (2009)

- Polio (2014). Da wurde ein internationaler Gesundheitsnotstand ausgerufen, weil die Krankheit wiederauflebte, obwohl sie vorher als nahezu ausgerottet galt.

- Ebola (2014)

- Zika (2016)

- Ebola (2018)

- COVID-19 (2019)

Bislang wurden alle internationalen Gesundheitsnotfälle durch Viren verursacht. Das müsste nicht so sein: Es könnten neben infektiösen Erregern beispielsweise auch chemische oder biochemische Waffen sein, die zur Ausrufung solcher Notstände führen.

Häufig trafen Epidemien die lokale Gesundheitsversorgung sowie die Forschungsgemeinschaft unvorbereitet. Für neu aufgetretene Erkrankungen, sogenannte Emerging Infections, wie beispielsweise SARS oder MERS und auch COVID-19 standen zum Zeitpunkt des Ausbruchs keine Impfstoffe zur Verfügung.

\section{Gamechanger: Ebola}

Der Ebola-Ausbruch 2014 bis 2016 in Westafrika, der über 11000 Menschen das Leben kostete, hat die Impfstoffentwicklung maßgeblich verändert. Denn unter dem Eindruck dieses Ausbruchs wurden Initiativen beschlossen, die auch bei der gegenwärtigen Entwicklung von Impfstoffen gegen COVID-19 sehr bedeutsam sind.

Einen solch großen Ebola-Ausbruch hatte man sich vorher kaum vorstellen können. So stand Ebola in den 1980-er Jahren bei den Experten sicher nicht oben auf der Liste der Infektionskrankheiten, gegen die man bevorzugt Impfstoffe entwickeln sollte. Dass es beim Ausbruch 2014 überhaupt potenzielle Impfstoffe gab, die getestet werden konnten, lag wohl auch daran, dass Ebola nach den Anschlägen auf das World Trade Center am 9. September 2001 auf der Liste der BioterrorismusWaffen erschien. Um die eigene Bevölkerung zu schützen, hatte es daher Förderung für entsprechende Forschung gegeben. Bis zum Ebola-Ausbruch 2014 war man jeder Epidemie mit der Impfstoff-Entwicklung hinterhergelaufen. Der Ausbruch markierte insofern einen Wendepunkt: Zum ersten Mal erfolgte die Impfstoff-Entwicklung in nie dagewesener Schnelligkeit noch während eines Ausbruchs. 
Die WHO hat 2017 eine Liste der damaligen Impfstoff-Kandidaten gegen Ebola veröffentlicht [3]. Diese Liste ist interessant, weil sich darauf einige Unternehmen, Konsortien und Impfstoff-Technologieplattformen wiederfinden, die heute auch bei der Impfstoff-Entwicklung gegen COVID-19 ganz vorne dabei sind. Außerdem zeigt die Liste, dass schon in dieser Epidemie in China und Russland Impfstoffe entwickelt und ohne das Durchlaufen der klassischen Impfstoffentwicklungsphasen zugelassen wurden. Das ist erwähnenswert, weil sich manche Menschen und Medien darüber empört haben, dass dort bereits COVID-19-Impfstoffe zugelassen sind.

Der erste zugelassene Ebola-Impfstoff war ein aktiv replizierender Vektor-Impfstoff, an dessen Entwicklung unsere Forschungsgruppe am Universitätsklinikum Hamburg-Eppendorf mitbeteiligt war. Es wäre wahrscheinlich gar nicht so einfach gewesen, diesen Vektor in einem anderen Kontext in die klinische Prüfung zu bringen. Die sehr schwierige Situation während des damaligen Ebola-Ausbruchs hatte somit auch eine gute Seite: Es steht ein neuer viraler Vektor zur Impfstoff-Entwicklung zur Verfügung.

Die Zulassung des Ebola-Vakzins Ervebo ${ }^{\circledR}$ erfolgte im November 2019. Angesichts dessen, dass der Ebola-Ausbruch 2014 begann, hat es also doch recht lange gedauert, bis der Impfstoff tatsächlich zugelassen wurde. Das ist insofern tragisch, weil jetzt gezeigt werden konnte, dass der Impfstoff eine über 97-prozentige Wirksamkeit hat. Mit einer Verfügbarkeit des Impfstoffes zu Beginn des Ausbruchs oder mehrere Monate früher in der Pandemie, hätte man den Verlauf der Pandemie wahrscheinlich maßgeblich beeinflussen können.

Aufgrund der COVID-19-Pandemie ist es weitgehend untergegangen, dass im Juli 2020 noch ein zweiter Ebola-Impfstoff zugelassen wurde. Er wird in zwei Einzeldosen verabreicht: Die erste Dosis beruht auf einem Adenovirus-Vektor (Zabdeno ${ }^{\circledR}$ ), die zweite auf einem sogenannten MVA-Vektor $\left(\right.$ Mvabea $\left.^{\circledR}\right)$. MVA steht für ModifiedVaccinia-Ankara-Virus (MVA) - es handelt sich dabei um ein abgeschwächtes Pockenvirus, das in seiner nicht rekombinanten Form auch als Pockenimpfstoff $\left(\right.$ Imvanex $^{\circledR}$ ) zugelassen ist. Bedeutsam ist, dass der verwendete Adenovirus-26-Vektor auch Grundlage eines COVID-19-Vakzins ist, das sich bereits in Studienphase 3 der Entwicklung befindet [4]. Die Erfahrungen aus der Ebola-Impfstoffentwicklung haben sich also nutzen lassen. Zum anderen erkennt man bei den zwei zugelassenen EbolaImpfstoffen, dass es unterschiedliche Impfregime gibt: Beim ersten Vakzin kommt man mit einer Dosis aus, während beim zweiten Vakzin zwei Dosen im Abstand von acht Wochen notwendig sind. Diese unterschiedlichen Impfregime existieren auch bei den Impfstoffen, die sich gegen COVID-19 in der Entwicklung befinden.

2016 formulierte Mimi Darko von der ghanaischen Lebensmittel- und Arzneimittelbehörde: „We should never again experience a crisis like the West Africa Ebola Epidemic. The world needs a more dynamic approach to Research and Development of live-saving drugs, vaccines and diagnostics." Sie formulierte damit sehr klar eine Auffassung, die sich damals in Politik und Wirtschaft durchzusetzen begann. Der westafrikanische Ebola-Ausbruch wurde zum Gamechanger, weil Initiativen auf den Weg gebracht wurden, um künftig Epidemien und Pandemien proaktiv bekämpfen zu können. So berief die WHO eine Experten-Kommission, die definiert hat, von welchen Infektionskrankheiten die größte Gefahr einer Pandemie ausgeht. Die entstandene Liste wird jährlich aktualisiert. 2018 umfasste sie folgende Krankheiten: 
- Krim-Kongo-Fieber

- Ebola und Marburg-Fieber

- Lassa-Fieber

- Middle East respiratory syndrome (MERS) und Severe Acute Respiratory Syndrome (SARS)

- Nipah and henipavirale Krankheiten

- Rift Valley Fieber (RVF)

- Zika

- Disease X

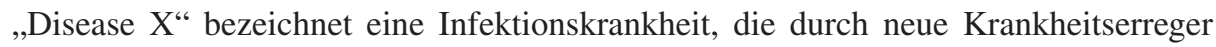
mit unbekannten Eigenschaften hervorgerufen wird. Für 2019 war COVID-19 diese Disease X. Auf der 2020-er Liste steht nun zusätzlich COVID-19 [5].

Zudem entstanden neue Initiativen mit dem Ziel, die Finanzierung für die Impfstoffentwicklung zu verbessern. An erster Stelle ist die „Coalition for Epidemic Preparedness Innovations“, kurz CEPI, zu nennen. Es handelt sich um eine globale öffentlich-private Non-for-profit-Partnerschaft von Staaten, Stiftungen, Forschungseinrichtungen und Pharma-Unternehmen. Finanziert wird sie unter anderem von Deutschland, Norwegen, Japan, Kanada, Australien, der Bill und Melinda Gates Stiftung und dem Wellcome Trust. Die CEPI hat die Entwicklung der meisten aussichtsreichen COVID-19-Impfstoffkandidaten finanziell unterstützt.

Die CEPI hat 2017 bei einer Präsentation das Bild einer künftigen Pandemie gezeichnet: „A virulent respiratory virus spreading as fast as flu could spread to all major global capitals within 60 days." Außerdem sagte die CEPI damals voraus, dass im schlimmsten Fall 33 Mio. Menschen innerhalb von 250 Tagen an der Krankheit sterben könnten, die von einem solchen Virus verursacht wird. Diese damalige Prognose macht deutlich, dass sich die Fachwelt durchaus mental auf eine Situation wie die der gegenwärtigen COVID-19-Pandemie vorbereitet hat. Dabei ist die gegenwärtige Lage, obwohl schlimm, bislang sogar weniger dramatisch, als es die CEPI damals befürchtet hat: Nach ungefähr 250 Tagen der Pandemie sind weltweit rund 1,2 Mio. Tote zu beklagen, bei 44 Mio. gemeldeten Infizierten (Stand: 29.10.2020). Allerdings ist die Pandemie noch nicht zu Ende und man kann die Situation daher nur vorläufig bewerten.

\section{Entwicklung von Impfstoffen gegen COVID-19}

Es ist einzigartig und verblüffend, wie kurz der Zeitraum von der Identifizierung des SARS-CoV-2-Virus bis zur ersten klinischen Prüfung war: Die Erkrankung trat im Dezember 2019 erstmals auf und im März 2020 wurde bereits die erste Testperson geimpft. Von der Entdeckung eines Erregers über die Sequenzermittlung bis zur Herstellung eines potenziellen Impfstoffs hat es also weniger als drei Monate gedauert. Auch die Zulassung des ersten Impfstoffs Ende 2020 ist ein unglaublicher Rekord, gemessen daran, wie lange es ansonsten von der Virenidentifizierung bis zur Impfstoffzulassung gedauert hat - oft 15 Jahre und mehr. 
Diese schnelle Entwicklung ruft in der Gesellschaft vor allem eine Frage hervor: Ist denn der Impfstoff trotz dieser Entwicklung sicher? Die Befürchtung, dass die Sicherheit unter der Schnelligkeit leidet, haben Kollegen vom Paul-Ehrlich-Institut in einem Artikel für das Ärzteblatt aufgegriffen [6]. Sie schreiben darin: „Das Paul-Ehrlich-Institut hat Maßnahmen ergriffen, um die Entwicklung und Zulassung von Impfstoffen zu beschleunigen, ohne dabei Kompromisse bei der Sicherheit einzugehen.“ Diese Botschaft gilt es, weiterzugeben. In Deutschland wird bei Impfstoffen die Verantwortung gegenüber der Bevölkerung sehr ernst genommen.

Auf den Internet-Seiten der WHO lässt sich stets aktuell erkennen, in welchem Stadium sich die verschiedenen Projekte zur Entwicklung von COVID-19-Impfstoffen gerade befinden [7]. Gestern, also am 29. Oktober 2020, gab es mehr als 170 VakzinKandidaten. Von denen befinden sich 45 bereits in der klinischen Prüfung, zehn davon in der Phase 3. In dieser letzten Phase der Prüfung wird festgestellt, ob jemand, der geimpft wurde, tatsächlich geschützt ist. Diese Daten fehlen uns derzeit noch, und wir sind sehr gespannt darauf.

Die drei Kandidaten, die als erstes über die Ziellinie gehen, sollen hier kurz einmal vorgestellt werden:

1. Ein Kandidat, von der CEPI gefördert, ist der von BioNTech und Pfizer. Es handelt sich um einen mRNA-Impfstoff. Er wird auch bei uns am Universitätsklinikum Hamburg-Eppendorf gemeinsam mit dem Bernhard-Nocht Clinical trial center (BNCCT) getestet: Diese Woche wurden 65 Probanden mit diesem Impfstoff geimpft. Es waren die letzten Probanden, die in Deutschland in die Phase-3-Studie eingeschlossen wurden. Deren Ergebnis wird mit Spannung erwartet.

2. Der zweite Kandidat ist ebenfalls ein mRNA-Impfstoff - und seine Entwicklung wurde ebenfalls von der CEPI finanziell unterstützt. Er stammt aus den USA, wo er in der Zusammenarbeit der National Institutes of Health (NIH), des National Institute of Allergy and Infectious Diseases (NIAID) und des Industriepartners Moderna entwickelt wurde. Im Juli 2020 stellten die Entwickler in einer einzigen Ausgabe eines Fachjournals zwei Studien vor: eine, bei denen der Impfstoff an Affen getestet wurde, und eine mit der Erprobung im Menschen. Das war auch ein einzigartiger Vorgang, denn normalerweise werden Tierstudien und klinische Studien streng nacheinander durchgeführt. Auch dieser Impfstoffkandidat hat bereits ein gutes Sicherheitsprofil gezeigt und gute Antikörper-Antworten.

3. Der dritte Kandidat ist ein viraler Vektorimpfstoff und beruht auf dem Schimpansen-Adenovirus, der auch bereits als Ebola-Impfstoff in klinischen Studien getestet wurde. Dieser Impfstoffkandidat, gefördert von CEPI, wurde ursprünglich von der Universität Oxford entwickelt, die inzwischen mit dem Unternehmen AstraZeneca zusammenarbeitet. Die Entwickler haben im Oktober eine Pressemitteilung herausgegeben, in der es heißt, dass das Vakzin auch bei älteren Menschen wirkt. Das ist bedeutsam, da ältere Menschen zu den Risikopatienten zählen, die besonders geschützt werden müssen.

In Deutschland hat sich das Bundesforschungsministerium in die Impfstoff-Entwicklung eingebracht und will zwei mRNA-Impfstoff-Projekte und ein Projekt zu einem viralen Vektor fördern. An den Projekten sind neben dem schon erwähnten BioNTech die zwei Unternehmen Curevac (Tübingen) und IDT Biologika beteiligt. 
Mit letzterem arbeitet unsere Gruppe des Universitätsklinikums Hamburg-Eppendorf zusammen.

Bei uns läuft derzeit eine Phase-1-Studie, die komplett aus öffentlichen Mitteln gefördert wird. An 30 Probanden wird vor allem die Sicherheit und Verträglichkeit des Impfstoffs erprobt. Bei dem Vektorimpfstoff wurde die genetische Information für ein Oberflächenprotein des COVID-19-Virus in ein inaktives Pockenvirus (MVA) eingebaut. Ein Team um Gerd Sutter von der Ludwig-Maximilians-Universität München und später ein Konsortium des Deutschen Zentrums für Infektionsforschung (DZIF) haben den Impfstoff gemeinsam entwickelt. Das Paul-Ehrlich-Institut hat die Studie am 30. September 2020 zugelassen. Am 7. Oktober ist die erste Probandin geimpft worden und $24 \mathrm{~h}$ die nächsten zwei Probanden und wiederum $24 \mathrm{~h}$ später die nächsten zwei. Dieses vorsichtige Vorgehen ist aus Sicherheitsgründen notwendig. Es ist also nicht so, dass alle 30 Probanden gleichzeitig die Impfung erhalten.

Inzwischen sind alle Probanden, die eine niedrige Dosis erhalten, das erste Mal geimpft. Nach der Datenanalyse und der Sicherheitsüberprüfung wird dann ab nächster Woche mit der Impfung der Gruppe begonnen, die eine höhere Dosis injiziert bekommt. Bisher läuft alles wie geplant (Stand 29. Oktober 2020).

Es stellt sich die Frage, ob denn angesichts von drei Vorreitern und über 170 Impfstoff-Konkurrenten die Mühe und der Aufwand für die Entwicklung und Erprobung des beschriebenen Vektorimpfstoffs überhaupt lohnt. Sicherlich werden nicht alle 170 Impfstoffe zugelassen. So viele Impfstoffe werden auch nicht benötigt. Aber die Situation ist eine einzigartige Gelegenheit für die Vakzinforschung: Es gibt sehr viele Phase-3-Studien und damit Wirksamkeitsprüfungen. Das wird es ermöglichen, besser $\mathrm{zu}$ verstehen, wie ein Impfstoff aufgebaut sein muss, damit von ihm eine Schutzwirkung ausgeht und welches die genauen Immunkorrelate für einen Impfschutz darstellen.

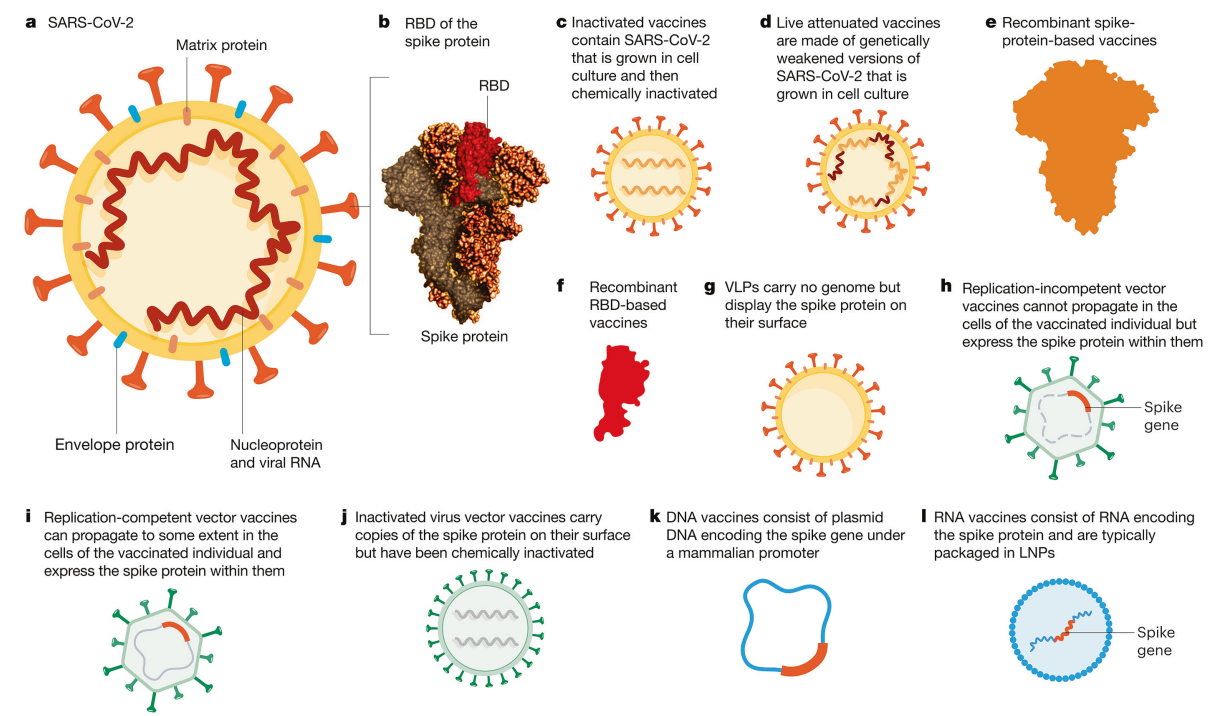

Abb. 1. Vakzin-Plattformen für die Entwicklung von COVID-19-Impfstoffen. Aus [8]. 
Es existieren sehr viele parallele Strategien zur Impfstoffentwicklung (Abb.1). Wer etwas tiefer in die Materie einsteigen will, dem sei sehr die Übersichtsarbeit empfohlen, aus der die Abbildung stammt [8]. Entscheidend ist, dass die Vakzinforschung nun die einmalige Gelegenheit hat, von der Empirie - ,Versuch und Irrtum“ - wegzukommen und die effektivsten Strategien zu ermitteln. Dabei helfen auch Impfstoffe, die nicht zur Zulassung kommen werden, weil die erhobenen Daten wertvolle Hinweise liefern, was eine Immunantwort auf einen Impfstoff verstärkt oder abschwächt. Mit einem solchen Wissen können noch bessere Impfstoffe generiert werden für die nächste Pandemie, die sicher kommen wird.

\section{Bedeutung von Impfstoffen}

Zum Schluss ist herauszustellen, was Impfstoffe eigentlich für die Menschheit bedeuten: Es gibt neben dem Zugang zu sauberem Trinkwasser keine Gesundheitsintervention, die die Sterblichkeit der Menschen auf der Erde mehr verringert hat als Impfstoffe. In Deutschland verdanken wir es den Impfstoffen, dass keine Frau im Wochenbett an Tetanus stirbt und dass wir keine Polio-Gelähmten mehr durch die Fußgängerzonen laufen sehen. Das wird bei vielen öffentlichen Diskussionen oft vergessen. Tatsächlich scheinen Impfstoffe manchmal ein Opfer ihres Erfolgs zu sein.

\section{Literatur}

1. Robert Koch Institut (RKI): Ausbrüche von Infektionskrankheiten. https://www.rki.de/DE/ Content/Infekt/Ausbrueche/Ausbrueche_node.html. Zugegriffen: 27. Jan 2021

2. https://www.who.int/ihr/procedures/pheic/en/. Zugegriffen: 16. Febr 2021

3. https://www.who.int/immunization/sage/meetings/2017/april/1_Ebola_vaccine_background_ document.pdf. Zugegriffen: 16. Febr 2021

4. https://www.janssen.com/emea/covid-19-vaccine. Zugegriffen: 16. Febr 2021

5. https://www.who.int/activities/prioritizing-diseases-for-research-and-development-inemergency-contexts. Zugegriffen: 16. Febr 2021

6. https://www.aerzteblatt.de/archiv/215884/COVID-19-Impfstoffentwicklung-Schneller-abersicher. Zugegriffen: 16. Febr 2021

7. https://www.who.int/publications/m/item/draft-landscape-of-covid-19-candidate-vaccines. Zugegriffen: 16. Febr 2021

8. Krammer, F.: SARS-CoV-2 vaccines in development. Nature 586, 516-527 (2020) 
Open Access Dieses Kapitel wird unter der Creative Commons Namensnennung - Nicht kommerziell - Keine Bearbeitung 4.0 International Lizenz (http://creativecommons.org/ licenses/by-nc-nd/4.0/deed.de) veröffentlicht, welche die nicht-kommerzielle Nutzung, Vervielfältigung, Verbreitung und Wiedergabe in jeglichem Medium und Format erlaubt, sofern Sie den/die ursprünglichen Autor(en) und die Quelle ordnungsgemäß nennen, einen Link zur Creative Commons Lizenz beifügen und angeben, ob Änderungen vorgenommen wurden. Die Lizenz gibt Ihnen nicht das Recht, bearbeitete oder sonst wie umgestaltete Fassungen dieses Werkes zu verbreiten oder öffentlich wiederzugeben.

Die in diesem Kapitel enthaltenen Bilder und sonstiges Drittmaterial unterliegen ebenfalls der genannten Creative Commons Lizenz, sofern sich aus der Abbildungslegende nichts anderes ergibt. Sofern das betreffende Material nicht unter der genannten Creative Commons Lizenz steht und die betreffende Handlung nicht nach gesetzlichen Vorschriften erlaubt ist, ist auch für die oben aufgeführten nicht-kommerziellen Weiterverwendungen des Materials die Einwilligung des jeweiligen Rechteinhabers einzuholen. 